\title{
Cláusulas abusivas entre empresas. Evolución en los principales sistemas de derecho comparado*
}

\author{
Unfair Terms Between Business. Evolution in the Main Systems of Comparative Law
}

Maria Elisa Morales Ortiz ${ }^{\text {a }}$

DOI: https://doi.org//10.11144/Javeriana.vj69.caee

Universidad Austral de Chile, Chile

maria.morales@uach.cl

ORCID: https://orcid.org/0000-0003-1200-7253

Recibido: 28 Octubre 2019

Pamela Andrea Mendoza Alonzo

Aceptado: 12 Noviembre 2019

Universidad Austral de Chile, Chile

ORCID: https://orcid.org/0000-0002-8396-2648

Renzo Esteban Munita Marambio

Universidad del Desarrollo, Chile

ORCID: https://orcid.org/0000-0003-2156-1416

\section{Resumen:}

Este artículo analiza la evolución que ha experimentado el control de contenido de cláusulas abusivas entre empresarios en los tres principales sistemas de derecho comparado. Los sistemas que se comparan son el inglés, alemán y francés, donde buscamos establecer si existe una tendencia, o si en cambio se verifican diferentes modelos. Finalmente, se concluye que, a través de diferentes técnicas legislativas, el control de cláusulas abusivas se extiende más allá de la relación de consumo a contratos entre empresarios, pero con una regulación distinta de la aplicable a los contratos de consumo.

Palabras clave: cláusulas abusivas, contratos entre empresarios, derecho del consumo, derecho comparado..

Abstract:

This article analyzes the evolution of the control of unfair terms' content among businesses in the main legal systems of comparative law. We compare the English, German, and French systems, looking to establish if a trend in Europe or different models exists. Finally, through different legislative techniques, it is concluded that unfair terms control extends far beyond the consumer contracts reaching $\mathrm{B} 2 \mathrm{~B}$ contracts, but according to separate and different regulations.

Keywords: Unfair Terms, Business to Business Contracts, Consumer Law, Comparative Law.

\section{Introducción}

En la teoría de las cláusulas abusivas existen, por lo menos, dos justificaciones para el control de su contenido ${ }^{1}$. La primera de ellas se refiere al abuso del poder de negociación y, una segunda justificación, relaciona la asimetría de información con los costos de transacción. De acuerdo con esta última, en la contratación por adhesión, cuando la parte adherente acepta los términos sin discutir, esto no se debe precisa o exclusivamente a su debilidad frente a su contraparte, sino que se trata de una decisión racional basada en el costo de discutir e intentar cambiar el contenido contractual. De ahí que, el control de cláusulas abusivas se extienda, además de relaciones $\mathrm{B}_{2} \mathrm{C}^{2}$, a relaciones $\mathrm{B}_{2} \mathrm{~B}^{3}$.

Este artículo se ha propuesto analizar el control de contenido de cláusulas abusivas entre empresarios en tres sistemas jurídicos de derecho europeo. Así, empleando la metodología de la "microcomparación” "se abordará el asunto específico del control de contenido de cláusulas abusivas entre empresarios en los denominados

Notas de autor

\footnotetext{
a Autor de correspondencia. Correo electrónico: maria.morales@uach.cl
} 
"sistemas padres": ordenamientos inglés, alemán y francés. El objetivo es determinar las similitudes y diferencias entre estos sistemas en dicho ámbito para, finalmente, establecer si se trata, o no, de un único modelo europeo.

Se intenta probar que en el derecho europeo el control de cláusulas abusivas también alcanza a aplicarse en relaciones entre empresarios, pero, en condiciones distintas que en la relación de consumo. Así, se intuye que los sistemas objeto de comparación emplean diferentes técnicas legislativas para extender el control de cláusulas abusivas a contratos entre empresarios, al sostener, en base a ello, que se trata de diferentes modelos.

Primero, analizaremos el control legal de contenido en cada sistema seleccionado, comenzando por el inglés, luego el alemán y terminando con el francés. En segundo lugar, realizaremos una micro comparación entre las soluciones de estos tres ordenamientos; $y$, finalmente, expondremos nuestras conclusiones.

\section{Derecho Inglés 6}

Antes de abordar el análisis de este sistema jurídico, cabe aclarar que los asuntos relativos al derecho comercial y al derecho del consumo en el derecho inglés se han desarrollado, especialmente, a través de leyes y no de decisiones de tribunales superiores. Por esta razón, en esta parte del trabajo nos referiremos preferentemente a aquella fuente del derecho.

\section{La situación previa a la implementación de la directiva 93/137}

Previo a la implementación de la Directiva 93/13, la regulación de cláusulas abusivas en el Reino Unido se encontraba únicamente en la Unfair Contract Terms Act (en adelante UCTA) de $1977^{8}$. Esta ley, vigente hasta ahora, pero con bastantes modificaciones, tiene por objeto regular las cláusulas contractuales de limitación o exoneración de responsabilidad. Por muchos años fue el único cuerpo normativo que estableció alguna clase de control de cláusulas abusivas en el Reino Unido hasta que, en el año 1994, se implementó la Directiva 93/13 por la Unfair Terms in Consumer Contracts Regulations (en adelante UTCCR) 9 .

Como Morales ha indicado ${ }^{10}$, la UTCCR no reformó ni derogó la normativa anterior a su entrada en vigor, por lo que ambas leyes coexistieron por más de 20 años, lo que produjo varios problemas de superposición normativa. La razón de lo anterior fue que la trasposición de esta directiva en el derecho inglés por la UTCCR se llevó a cabo a través de una copia directa, sin mediar un proceso de adaptación del derecho interno ${ }^{11}$. Esta técnica de implementación fue bastante criticada ${ }^{12}$, no obstante, la UTCCR ha marcado un hito en el derecho contractual inglés y ha sido catalogada como "la legislación más importante en el ámbito del derecho contractual" ${ }^{13}$ de dicho ordenamiento.

En ese ambiente de coexistencia y superposición normativa había ciertas diferencias fundamentales entre ambos cuerpos normativos que, para efectos de este artículo, conviene hacer notar. En primer lugar, la UCTA se aplicaba tanto a cláusulas libremente discutidas como a cláusulas no negociadas en tanto que, la UTCCR, sólo se aplicaba a cláusula no negociadas. En segundo lugar, en cuanto al concepto de consumidor, para la UCTA es quien celebra el contrato fuera de curso de su negocio teniendo como contraparte a quien lo hace dentro del curso del suyo, sin excluir a las empresas, en tanto que para la UTCCR se trataba de toda persona natural que actúa con un propósito ajeno a su negocio, empresa o profesión. En tercer lugar, la UCTA resultaba aplicable a contratos de consumo (B2C) y a contratos entre empresas (B2B) hasta el año 2015, en tanto que la UTCCR era aplicable sólo a contratos B2C. La UCTA no contiene ni una definición ni una lista de cláusulas abusivas, pero sí un test de razonabilidad al que se debe someter la cláusula respectiva; por su parte, la UTCCR sí definía cláusula abusiva y contenía una lista gris de cláusulas que podrían ser consideradas abusivas. 
La situación de superposición antes aludida se resolvió con la dictación de una nueva ley, la Consumer Rights Act 2015 (CRA) ${ }^{14}$, que reemplaza parcialmente a UCTA y totalmente a la UTCCR, convirtiéndose en la nueva ley que implementa la Directiva 93/13. Las disposiciones de UCTA sobre contratos B2C fueron reemplazadas por CRA, subsistiendo en lo demás (contracts B2B y C2C). La UTCCR fue reemplazada completamente por CRA.

\section{Regulación actual de las cláusulas abusivas entre empresarios en el derecho inglés}

De acuerdo con la regulación actualmente vigente, el control de cláusulas abusivas en el Reino Unido opera a través de dos leyes con distinto ámbito de aplicación. CRA, para contratos de consumo y UCTA para contratos B2B.

De acuerdo con la primera de ellas, el diseño del control comprende una definición de cláusulas abusivas ${ }^{15}$, una test de abusividad ${ }^{16}$, y una lista gris de cláusulas que pueden ser declaradas abusivas. ${ }^{17}$ Dentro de ese esquema, la regla general es que algunas cla\#usulas pueden someterse al test de abusividad, otras deben ser consideradas abusivas (las que inviertan a carga el test en perjuicio del consumidor) ${ }^{18}$, otras quedan automa\#ticamente sin efecto (las que excluyen o restringen la responsabilidad por muerte o lesiones) ${ }^{19} \mathrm{y}$ otras quedan, en principio, excluidas del test de abusividad en la medida que cumplan con el principio de transparencia ${ }^{20}$.

Por último, es relevante notar que bajo la CRA 2015 se entiende como consumidor, a aquel individuo (persona natural) que actúa con fines total o principalmente fuera de su negocio, empresa, oficio o profesión ${ }^{21}$. Sobre esta base, no es posible considerar bajo la designación de consumidor a empresas, sin importar su tamaño.

Con lo anterior, en cuanto a cláusulas abusivas entre empresarios, la situación vigente quedó regulada exclusivamente por la UCTA. Aunque su título sugiere un gran campo de control de cláusulas abusivas, lo cierto es que ese espectro no es tal, pues tiene una restricción: solo se aplica a cláusulas de exoneración o limitación de responsabilidad. No obstante, se aplica tanto a cláusulas libremente negociadas como a cláusulas no negociadas.

\section{El Test de abusividad de la UCTA 1977 y los criterios que sirven de guía para su aplicación}

En el marco de la UCTA 1977, el control de contenido de las cláusulas se lleva a cabo a través de un test de razonabilidad o justicia. Sin embargo, no todas las cláusulas se someten a esta. En efecto, ciertas cláusulas quedan automáticamente sin efecto: aquellas que pretenden excluir la responsabilidad del proveedor por muerte o lesiones debido a su negligencia. Todas las demás cláusulas pueden ser consideradas válidas si cumplen con el requerimiento justicia (reasonableness).

Cabe especificar que la UCTA, en cuanto a su estructura, consta de 3 partes ${ }^{22}$ y 4 anexos. Esto es relevante porque la regulación en cada parte presenta algunas diferencias en el tratamiento del tema y uno de sus anexos especifica una guía con algunos criterios orientadores. La tercera parte de la ley, que se aplica a todo el Reino Unido, contiene disposiciones generales y relativas a contratos internacionales, por lo que no nos referiremos a ella.

La primera parte de la ley se aplica a Inglaterra, Gales e Irlanda del Norte y se extiende desde la sección 1 a la 14. En esta parte el test de justicia se encuentra establecida en la sección 11 y su objeto es evaluar el equilibrio de los derechos y obligaciones de las partes del contrato. Para estos efectos, se deben tener en cuenta las circunstancias del momento de la celebración del contrato y si las cláusulas en cuestión fueron razonablemente conocidas o consideradas por las partes en dicho momento. En la aplicación del test se deben 
tener en cuenta, particularmente, los criterios establecidos en el anexo número dos de la ley ${ }^{23}$ : a) el poder negociador de las partes, considerando, entre otras cosas, si el cliente (customer) tuvo algún medio o forma alternativa de cumplir con sus requerimientos; b) si el cliente recibió un incentivo para aceptar la cláusula, o si tuvo la oportunidad de celebrar un contrato similar con otra persona, sin la cláusula en cuestión; c) si el cliente sabía o debería haber sabido razonablemente sobre la existencia y el alcance del término; d) si la cláusula excluye o restringe la responsabilidad por incumplimiento de un término contractual cuando era razonable, al tiempo del contrato, considerar viable su cumplimiento; e) si los bienes fueron fabricados, procesados o adaptados al pedido especial del cliente.

La segunda parte de la $\mathrm{UCTA}^{24}$ se aplica a Escocia. Esta parte se extiende desde la sección 15 a la 25. En esta parte, el test de justicia ${ }^{25}$ es muy similar al establecido en la primera parte, pero agrega que, cuando una cláusula pretenda restringir la responsabilidad a una suma específica de dinero, se debe tener en cuenta particularmente: a) los medios que la parte que busca beneficiarse de la cláusula tendría a su disposición en caso de tener que cumplir con su responsabilidad; $y, b$ ) hasta qué punto era viable para esta misma parte cubrir su responsabilidad con un seguro. Por último, esta parte de la ley establece expresamente que la carga de probar que el término contractual es justo recae sobre quien así lo alega.

\section{Derecho alemán}

\section{La evolución de la regulación de cláusulas abusivas en el derecho alemán}

Alemania ha sido uno de los países europeos que ha ido a la vanguardia en la regulación de condiciones generales de la contratación y control de las cláusulas abusivas, caracterizándose también por tener un sistema de control judicial de cláusulas bastante estricto comparativamente ${ }^{26}$.

Efectivamente, ya en el año 1976 entra en vigor la Ley sobre términos y condiciones generales (Gesetz zur Regelung des Rechtsder Allgemeinen Geschäftsbedingungen, AGBG). Sin embargo, no se trata estrictamente de una Ley de protección al consumidor. Se trata de una ley que disciplinó especialmente los términos generales del contrato, al establecer un catálogo de cláusulas abusivas, recogidas de una larga tradición jurisprudencial, aplicables cada vez que exista una parte débil en la relación contractual ${ }^{27}$. Dichos criterios jurisprudenciales se desarrollaron inicialmente en torno a la noción de buenas costumbres del apartado 1 del artículo $138^{28}$ del Bürgerliches Gesetzbuch $\left(\mathrm{BGB}^{29}\right)$, para posteriormente ampliarlo, a partir de los años 50, a los estándares de la buena fe contractual del artículo 242 del BGB ${ }^{30}$.

Posteriormente, con el fin de comenzar a armonizar el derecho interno con la Directiva 93/13, la AGBG fue reformada el 19 de julio de 1996 (Gesetz zur Änderung des AGB-Gesetzes und der Insolvenzordnung). En todo caso, es sabido que la AGBG sirvió en parte de inspiración en la redacción de esta directiva, por lo que en dicha reforma destaca sólo la modificación del artículo 12 (Internationaler Geltungsbereich) y un nuevo artículo, el artículo 24a (Verbraucherverträge), relativo a los "contratos de consumo", fortaleciendo así la protección al consumidor por sobre otros contratantes débiles ${ }^{31}$.

De todas formas, el AGBG siguió siendo una ley de condiciones generales de la contratación de aplicación general $^{32}$, tendencia que ha continuado con las reformas al BGB del año 2000 (que incorporó los $\$ \$ 13-14$ ) y del $2001^{33}$ (que derogó la AGBG y la incorporó en los $\$ \$ 305-310^{34}$.) respectivamente. Ambas reformas, terminaron de adaptar, aunque sin grandes modificaciones, el derecho nacional en este tema en relación con la Directiva 93/13. Así, actualmente los artículos 305-310 del BGB ${ }^{35}$ son las normas referentes en este tema, tal como en su momento lo fue la $\mathrm{AGBG}^{36}$. 


\section{Extensión de la regulación sobre cláusulas abusivas a contratos b2b en el derecho alemán}

Como se dijo, la regulación sobre los términos y condiciones alemana ( $A G B$-Recht) fue pensada tanto para aplicarse a las relaciones de consumo, como entre empresarios ${ }^{37}$. En la misma línea continuó el BGB, que en una reforma previa a la del 2001, del 27 de junio de 2000 (Gesetz über Fernabsatzverträge und andere Fragen des Verbraucherrechts sowie zur Umstellung von Vorschriften auf Euro) incorporó una definición de consumidor (Verbraucher) en el artículo $13^{38}$ y de empresario (Unternehmer) en el artículo $14^{39}$. En dicho sentido, la expresión Unternehmer es bastante amplia, por lo que en principio no cabe hacer una distinción entre grandes y pequeños-medianos empresarios ${ }^{40}$. No obstante, la protección conferida al empresario adherente formalmente es menor que en el caso de los contratos B2C, ello según el mismo tenor del artículo 310, que excluye de la "lista gris" ( $\mathrm{N}^{\circ}$ s. 1, 2 a 8) del artículo 308 y de la "lista negra" del artículo 309 a los contratos B2B. Asimismo, reserva también la aplicación de los apartados 2 y 3 del artículo 305. Sin embargo, esta medida ha tenido efectos más bien limitados, pues la jurisprudencia de todas formas utiliza dichas listas para determinar la mala fe en un contrato empresarios, lo que las hace aplicables indirectamente en la práctica en esas situaciones ${ }^{41}$. En dicho sentido, cobra especial fuerza el artículo 307 en la configuración de la protección al empresario más débil en relaciones $\mathrm{B}_{2} \mathrm{~B}^{42}$, estableciéndose un control de contenido general a través de su aplicación. Así, el mandato de "transparencia" consagrado en el artículo 307 (un perjuicio indebido puede resultar también cuando la cláusula esté redactada de forma no clara e incomprensible) es frecuentemente utilizado como principio básico de las condiciones generales de la contratación y donde las relaciones $\mathrm{B} 2 \mathrm{~B}$ no son la excepción ${ }^{43}$. También destaca la tendencia jurisprudencial proveniente de la interpretación del AGBG, en el que de todas formas se hacía una interpretación extensiva de los artículo 10-11 (hoy artículo 308-309). Efectivamente, el Tribunal Federal de Justicia de Alemania (Bundesgerichthof, BGH) justifica dicha interpretación en la llamada "eficacia indiciaria" (Indizwirkung) ${ }^{44}$ de ese listado de cláusulas abusivas. Indizwirkung se traduce en lo siguiente: si la cláusula incorporada en el contrato B2B se encuentra bajo uno de los supuestos del artículo 309 (principalmente), es un "indicio" de que también el empresario adherente se encuentra bajo un desequilibrio, a menos que se enmarque en los intereses y necesidades del tráfico mercantil ${ }^{45}$.

De todas formas, la jurisprudencia alemana ha hecho distinciones a la hora de aplicar estas interpretaciones, haciendo una diferenciación entre tipos de empresarios ajena al artículo 14 del BGB. Así, aumenta la protección al pequeño empresario, matizando hasta excluir a las grandes empresas ${ }^{46}$. Este y otros aspectos han sido objeto de críticas.

En efecto, la interpretación jurisprudencial no ha tenido una plena acogida en todos los sectores, hay voces que piden que existan niveles de protección diferentes tratándose de las relaciones entre empresarios ${ }^{47}$. Así, por tratarse de relaciones jurídicas distintas, la aplicación indirecta de los artículos 308-309 a través del artículo 307 elimina la especialidad de ellas mismas ${ }^{48}$. En este sentido, se reafirma esta necesidad de distinción puesto que lo que provoca esta regulación e interpretación judicial demasiado estricta es el éxodo de los empresarios hacia legislaciones que admiten mayor flexibilidad ${ }^{49}$.

Por otro lado, el hecho de que la jurisprudencia haga una distinción en la práctica entre tipos de empresarios cuando la ley no lo distingue, también genera reproches ${ }^{50}$. Así, entre otros argumentos, se dice que la protección del empresario "débil" frente al "fuerte" no es una labor que corresponda definir en la regulación de condiciones generales, sino al derecho de la competencia ${ }^{51}$. 


\section{Derecho francés}

\section{Evolución y contexto del control de cláusulas abusivas en el derecho francés}

En Francia el control de cláusulas abusivas data de mucho antes de la dictación de la Directiva 93/13 $3^{52}$. De hecho, la cuestión de la implementación de esta directiva fue objeto de debate $e^{53}$, llegando a considerarse incluso innecesaria bajo el argumento de que la legislación francesa, vigente en ese entonces, ya era compatible con los requisitos de la norma europea ${ }^{54}$. Además, existían otras resistencias, tales como a la lista indicativa de cláusulas que pueden ser declaradas abusivas, contenida en el anexo de la directiva, ámbito en el cual ya se contaba desde antes con una lista de recomendaciones de la Commission des clauses abusives ${ }^{55}$ que, aunque no vinculantes, se superponían a varias de las disposiciones del mencionado anexo ${ }^{56}$.

Por otro lado, se criticaba la definición general de cláusula abusiva contenida en el artículo 3 de la directiva ${ }^{57}$ calificándola de imprecisa y como una fuente de inseguridad jurídica ${ }^{58}$. Así, tampoco se creyó necesario incorporar la referencia a la buena fe que allí se contiene ${ }^{59}$. Con todo, el derecho francés finalmente se modificó en aras de la adecuación al derecho comunitario con la promulgación de la Ley 95-96 del 1. ${ }^{\circ} \mathrm{de}$ febrero de $1995^{60}$ que conservó algunos elementos vigentes e incorporó otros nuevos. Luego, esa legislación fue integrada en 1995 en el Code de la Consommation. Este desarrollo previo ha dado a las normas contra cláusulas abusivas una fisonomía propia. En efecto, en el derecho francés se detecta una omnicomprensión en el tratamiento de las cláusulas abusivas, consecuencia de un fenómeno verificado a mediados de la segunda mitad del siglo pasado, época en que se percibe "la idea que una desigualdad podía también nacer de la existencia de ciertas cláusulas que, otorgando prerrogativas excesivas a una de las partes, provocan un desequilibrio de la convención" ${ }^{61}$.

Dentro de dicho ámbito, no solo son comprendidas aquellas que tienen por objeto proteger consumidores o no profesionales que contratan con un profesional, sino que también pueden tener lugar en contratos $\mathrm{B} 2 \mathrm{~B}^{62}$, por cuanto "decidir de una forma distinta, significaría satisfacer una protección selectiva y discriminatoria (en favor del consumidor)" ${ }^{\prime 3}$. Así las cosas, la protección según da cuenta en entramado jurídico francés es amplia, asunto que será objeto de análisis en los párrafos que siguen.

\section{Control legal de contenido de cláusulas abusivas en el derecho francés: el entramado legislativo}

El recientemente reformado art. L. 442-6, I, 2. ${ }^{\circ}$ del Código de Comercio, fruto de la ley de 4 de agosto de $2008^{64}$, extendió la tutela al mundo contractual B2B ${ }^{65}$. Sin embargo, la indicada norma no es, sino que reflejo de "una etapa suplementaria en el encuadre de la redacción de contratos comerciales con el propósito de proteger a la parte débil" ${ }^{66}$. Ello, pues desde la ley del 10 de junio de 1978, que incorporó el tratamiento de la cláusula abusiva en Francia, comenzaron a verificarse manifestaciones concretas en el sentido de lo expuesto. De ahí que el art. L. 212-1 del Código del Consumo (antiguo artículo L. 132-1 ${ }^{67}$ ) concretice el amparo, por una parte, a los contratos B2C; mientras que, por la otra, y de acuerdo con el art. L. 212-2 del mismo Código, también se proteja a no profesionales en contratos celebrados con profesionales. Respecto de la sanción en ambos casos, una vez que la cláusula se estima como abusiva de conformidad a la ley ${ }^{68}$, esta "se tiene por no escrita" ${ }^{69}$, sin que la validez del contrato en si misma se vea afectada. Más allá de lo anterior, el nuevo art. 1171 del $\operatorname{Code}^{70}$, sanciona en términos generales, las cláusulas abusivas de contratos de adhesión celebrados con posterioridad al 1 de octubre de 2016, con independencia de la calificación de las partes del contrato. 
Luego entonces, perfectamente un profesional puede impugnar una cláusula desproporcionada de un contrato suscrito con otro profesional, sin tener la necesidad de encasillar su acción dentro de la norma de derecho comercial ${ }^{71}$. La incorporación de esta norma es muy relevante, pues "no existía en el derecho común de los contratos, reglas habilitantes a rechazar la aplicación de una cláusula considerada como abusiva. Si no era por la vía de la existencia de un vicio del consentimiento o de una ausencia de voluntad el contrato no podía ser cuestionado cuando una o varias cláusulas parecieran abusivas (...) en este derecho común podía también evocarse la teoría de la causa, hoy suprimida por la ordenanza del 2016 "72.

El devenir legislativo no acabó aquí, por cuanto la reciente Ordenanza n. ${ }^{\circ} 2018-359$ de 24 de abril de 2019, que reformó el título IV del libro IV del Código de Comercio, y en este entendido, alteró el mencionado art. L. 442-6, reescribiéndolo entre los nuevos arts. L. 442-1 al L. 442-4, que cobijan una técnica dispositiva lo suficientemente amplia como para entender comprendido dentro de su órbita a profesionales afectados por una desproporción significativa, derivada de un contrato celebrado con otro profesional. En lo medular, la norma que nos interesa es el nuevo art. L. 442-1, I, 2. ${ }^{\circ}$ que, actualmente, dispone:

\footnotetext{
"Compromete la responsabilidad de su autor y lo obliga a reparar el daño causado, el hecho cometido en el cuadro de la negociación comercial, de la conclusión o de la ejecución del contrato, por toda persona que ejerce actividades de producción, de distribución o de servicios, (Relativo a): $2^{\circ}$ : Someter o intentar someter a la otra parte a obligaciones que creen un desequilibrio significativo en los derechos y obligaciones de las partes".
}

Es del caso destacar que el legislador se refiere a "obligaciones que creen un desequilibrio significativo", sin vincularla a un tipo contractual específico ${ }^{73}$. La ley, además, no se refirió a materias en las que no podía entenderse que la cláusula era abusiva, bastando acreditar el sometimiento o el intento de someter ${ }^{74}$, mientras que el desequilibrio significativo ${ }^{75}$ al cual también hacía referencia la antigua norma, si bien podía dar lugar a una acción de reparación de daños, vedaba a la víctima, al menos formalmente, a impetrar la nulidad de las cláusulas en las que radicaba la desproporción ${ }^{76}$.

Esta modificación al Código de Comercio plantea la disyuntiva de determinar la pertinencia de un ejercicio concurrente o alternativo del hoy reformado art. L. 442-6, I, $2^{\circ}$ con el art. 1171 del Code $e^{77}$, frente a un supuesto de abuso. No existe claridad sobre cuál es la línea divisoria de los campos de aplicabilidad de los citados artículos, ni sobre cuáles son sus puntos de distinción.

Como vimos, el art. 1171 del Code, ajusta la protección exclusivamente al ámbito de los contratos de adhesión. Además, la norma se refiere a que la cláusula abusiva se tendrá por no escrita, sin excluir la posibilidad de demandar una indemnización de daños. Es del caso mencionar, además, que la disposición debe leerse en armonía con el reconocimiento positivo que goza el contrato en referencia, el cual no quedó al margen de la última gran reforma al derecho de los contratos y del test de las obligaciones en Francia. Ella se verificó debido a la Ley n. ${ }^{\circ} 2018-287$ del 20 de abril de 2018, que ratificó la ordenanza n. ${ }^{\circ} 2016-131$ del 10 de febrero de 2016. En este sentido, dentro de las normas que incorpora dicha mise a jour, es la conceptualización del contrato de adhesión, entendiéndolo como: "aquel que comporta un conjunto de cláusulas no negociables, determinadas anticipadamente por una de las partes”, por el art. 1110 inciso $2^{\circ}$.

De acuerdo con lo anterior, cabe preguntarse: ¿es del caso entonces proyectar aquí un supuesto de aplicación del principio de la especialidad? Hacemos presente que dicho principio fue consignado expresamente por el Código Civil en el art. 1105, tras la ordenanza de 2016. Entonces, habría que tener en cuenta que al ser una norma especial la del Código de Comercio, aquella del Código Civil no tendría posibilidad de ser invocada, cuando el asunto tenga relación con una cláusula abusiva fijada en el marco de una "negociación comercial". Sin perjuicio de lo anterior, Larroumet y Bros opinan que:

\footnotetext{
"si un contrato celebrado entre comerciantes es un contrato de adhesión, lo que es frecuente, en el cual fue estipulada una cláusula abusiva, no hay razón de prohibir a la víctima del abuso de demandar el sancionamiento esta cláusula bajo el fundamento del art. 1171 del Código Civil" 78 .
} 
Lo que mencionamos se funda en que no viene al caso proyectar entre ambas normas un conflicto de leyes que deba resolverse en atención a la identificación de una ley general o de otra especial. En rigor, el problema se presenta respecto de normas representativas de un mismo espíritu o persecutoras de un mismo objetivo, lo que no se da en los hechos. En sentido de lo anterior:

"Contrariamente al desequilibrio significativo del Código de Comercio, que debería obedecer a las especificidades particulares en razón de su objetivo, esto es, la prohibición de estipulaciones restrictivas de competencia, el desequilibrio significativo del Código Civil, independientemente de su dominio de aplicación respecto de personas y de contratos, corresponde al del Código del Consumo"79.

De conformidad con lo anterior se propusieron dos lecturas en la articulación de ambas normas: por una parte, un ejercicio opcional; y por otra, uno de carácter acumulativo. Desde la lectura del primero, podría convenir el ejercicio de la acción bajo la órbita del art. 1171, toda vez que a través de él no hay plazo de prescripción que afecte el que se tenga por no escrita la cláusula, pues dicho efecto implica la inexistencia de ella. Respecto del segundo, el razonamiento va en la línea de admitir que un empresario que suscribió un contrato de adhesión, y que a su vez se ha visto afectado por una cláusula abusiva, pueda invocar el artículo 1171, con el propósito que esta cláusula se tenga por no escrita, pidiendo una indemnización de daños bajo el argumento de la norma del estatuto comercial ${ }^{80}$.

Con todo, bajo el imperio de la norma recientemente aprobada, la utilidad en la invocación de la norma de derecho común, por sobre la de derecho comercial, se ha reducido. Ello no es sino una consecuencia de la posibilidad de obtener la nulidad de la cláusula abusiva también en el capítulo comercialista, según referimos. La ausencia de esta prerrogativa de la víctima era lo que motivaba a la doctrina a desvirtuar los argumentos que se proyectaban en la línea del principio de especialidad ya reseñados. Más allá de lo anterior, aún pervive un argumento de no poca importancia en cuanto a la inclinación de fundar la acción en el Código Civil. Este argumento va en la línea de intentar soslayar las limitaciones de carácter territorial, así como del tribunal competente para conocer del ejercicio de la acción, aspectos que pueden significar un escollo para el afectado.

\section{Microcomparación: semejanzas y diferencias de las soluciones}

Luego de haber analizado cómo ha evolucionado y cómo se plantea el control de contenido de cláusulas abusivas entre empresarios en los "sistemas padres" actualmente, corresponde ahora realizar el análisis comparativo. A pesar de que las diferencias son lo primero que el comparatista nota al enfrentarse al estudio de las normas de otro ordenamiento ${ }^{81}$, no desconoceremos la existencia de puntos en común. Por esa razón, la comparación se planteará no solo en términos de contraste, sino que también pondremos de relieve las similitudes entre las soluciones de los tres ordenamientos ${ }^{82}$.

De la comparación entre los tres sistemas seleccionados, las similitudes más significativas entre ellos son las siguientes. Primero, los tres sistemas han dispuesto control legal de contenido de cláusulas abusivas antes de la dictación de la Directiva 93/13, tanto para relaciones entre consumidores como para relaciones entre empresarios. Segundo, en los tres sistemas el control de contenido de cláusulas abusivas entre empresarios difiere del control relativo a las relaciones de consumo, en cuanto a la técnica legislativa empleada. En otras palabras, el control legal de contenido que se aplica a las relaciones de consumo B2C, no es el mismo control legal de contenido que se aplica a las relaciones B2B. Y, tercero, en conformidad a lo anterior, en los tres sistemas, el aparato legislativo aplicable al tratamiento de la abusividad de cláusulas se proyecta desde la perspectiva de la calificación jurídica de las partes contratantes.

Luego, del ejercicio comparativo entre los tres ordenamientos seleccionados surgen — sin perjuicio de otras - las siguientes diferencias relevantes para este trabajo. Primero, los sistemas padres han empleado distintas técnicas legislativas al establecer el control de cláusulas abusivas entre empresarios. En síntesis: en el sistema 
inglés, este control se establece en una ley especial con un ámbito material restringido, se aplica a través de un test de razonabilidad tanto a contratos libremente discutidos como a contratos por adhesión, con apoyo en unos criterios de justicia establecidos en la misma ley. En el derecho alemán, el control tanto para relaciones B2C como B2B se dispone en el Código Civil, pero en el caso de estas últimás, con un umbral de protección menor, pues la lista negra y gris, no son aplicables a empresarios, no obstante, la interpretación jurisprudencial extensiva. Por último, en el derecho francés el control está dispuesto en el Código de Comercio y en el Código Civil, la diferencia es que en el primero la técnica resulta ser más generosa que en el segundo, toda vez que no requiere de contratos de adhesión, ni tampoco se contemplan limitaciones a declaración de abusividad de la cláusula, tal como se aprecia en el Código Civil. Una segunda gran diferencia es que de los tres sistemas solo uno de ellos -el ordenamiento inglés - establece una lista de criterios específicos que pueden servir de guía al juez en la determinación de si una cláusula puede ser declarada abusiva en las relaciones entre empresarios.

\section{Conclusiones}

En síntesis, el Derecho inglés no aplica el control de contenido resultante de la implementación de la Directiva 93/13 a las relaciones contractuales entre empresarios, sino que controla cláusulas abusivas entre empresarios a través de una ley especial, diferente y preexistente a dicha norma europea. Este estatuto especial es la UCTA 1977. Las diferencias más importantes entre el control de contenido de la CRA 2015 —referida a relaciones de consumo- y la UCTA 1977 son, en primer lugar, que esta última tiene un ámbito de aplicación objetivo especial ya que el test de justicia recae sobre una clase específica de cláusulas que son aquellas destinadas a limitar o a exonerar de responsabilidad a una de las partes. En segundo lugar, el control en las relaciones entre empresarios se extiende tanto a cláusulas negociadas como a cláusulas predispuestas. Por último, la técnica de control es diferente ya que se establece un test de justicia de acuerdo con unos criterios que guían al juez en la determinación de abusividad.

En lo que respecta al derecho alemán, este sistema puede verse como un buen ejemplo de modelo de protección a las Pymes y ha servido de guía para otros derechos nacionales y Derecho comunitario europeo. Así, al contrario de lo que sucede en otros ordenamientos, la discusión actual no se centra en si las relaciones $\mathrm{B} 2 \mathrm{~B}$ pueden ser objeto de control de contenido, sino en si es necesario o no retroceder en esa protección. En dicho sentido, no se debe desconocer la fuerte tradición jurisprudencial protectora del empresario más débil - ya desde antes de la dictación del AGBG - lo que hace que las propuestas de reforma al BGB (de materializarse) puedan resultar inoficiosas en la práctica.

En el derecho francés, al menos en cuanto a la protección de consumidores, el tratamiento aplicable a cláusulas es también anterior a la Directiva 93/13, incluso con un interesante desarrollo jurisprudencial. Luego de la implementación de la citada directiva, la actividad legislativa apoyada por estudios de doctrina extendió la protección al espectro B2B, tanto en el año 2008, como en abril de 2019. Aunque el control legal de cláusulas abusivas se aloja en el Código de Comercio, también en el Código Civil existen normas aplicables a relaciones B2B. Sin embargo, la abusividad solo puede ser revisada respecto de contratos de adhesión, considerándose una expresa exclusión a las cláusulas abusivas respecto del precio como de los elementos esenciales del contrato.

Finalmente, hemos podido determinar que no existe un único modelo de control de cláusulas abusivas en el derecho europeo. Del análisis de la evolución y regulación actual en los llamados "sistemas padres" fluye que estos ordenamientos coinciden en la extensión del control de cláusula abusivas a relaciones $\mathrm{B} 2 \mathrm{~B}$, pero con distinta técnica legislativa. Esto nos permite concluir que existen, al menos, tres modelos distintos de control de cláusulas abusivas entre empresarios. 


\section{Bibliografía}

Antonia Sommerfeld, Rechtsflucht ins Ausland wegen des deutschen AGB-Rechts im B2B-Verkehr, 11 RIW-Recht der internationalen Wirtschaft, n. ${ }^{\circ}$ 15, 741-747 (2018).

B. Gsell, Deutsche erfahrungen mit der begrenzten erstreckung der klauselkontrolle auf den unternehmerischen verkehr, en Standardisierte Verträge - zwischen Privatautonomie und rechtlicher Kontrolle, 237-258 (Johann Kindl, María Pilar Perales Viscasillas \& Tatiana Arroyo eds., Standardisierte Verträge-zwischen Privatautonomie "und rechtlicher Kontrolle, 2017).

Bürgerliches Gesetzbuch (BGB), Reichsgesetzblatt n. ${ }^{\circ} 21,18$ de agosto de 1896 (Alemania).

Camille Dupin, La notion de consommateur, (2013-2014), (Mémoire Master Droit des Contrats, Université Pris). htt p://memoire.jm.u-psud.fr/affiche_memoire.php?fich=3975\&diff=public

Christoph Schmith \& Detlef Ulmer, Allgemeine Geschäftsbedingungen und Verträge für Unternehmen: Chancen und Risiken (Springer, 2010). https://doi.org/10.1007/978-3-642-01475-8

Claire-Marie Peglion-Zika, La notion de clause abusive au sens de l'article L. 132-1 du Code de la consommation (2013) (tesis doctoral, Université Panthéon-Assas). https://docassas.u-paris2.fr/nuxeo/site/esupversions/7b78847a-d 9e6-4ed4-8d21-fabc0c1afbf8?inline

Código de Consumo (Code de la Consommation), 13 de noviembre de 2020. https://www.legifrance.gouv.fr/codes /texte_lc/LEGITEXT000006069565/

Commission des Clauses Abusives. Recommandation de synthèse n. ${ }^{\circ} 91-02$ relative à certaines clauses insérées dans les contrats conclus entre professionnels et non-professionnels ou consommateurs (BOCCRF du 6/09/1991).

Consumer Rights Act. 2015 c. 15. TSO. 26 marzo 2015 (Reino Unido).

Corte de Apelaciones de París en fallo de 30 de mayo de 2017, JurisData: 2017-010882 (Francia).

Corte de Apelaciones de París, JurisData: 2017-010849. 17 de mayo de 2017 (Francia).

Cyril Grimaldi, Les limites à la libre détermination du contenu du contrat dans le nouveau droit des contrats, Lextenso étudiant, (10 de noviembre, 2016). https://etudiant.lextenso.fr/article-\%C3\%A0-la-une-contrats/les-limites-\% C3\%A0-la-libre-d\%C3\%A9termination-du-contenu-du-contrat-dans-le-nouveau

Elizabeth Macdonald, Scope and Fairness of the Unfair Terms in Consumer Contracts Regulations: Director General of Fair-Trading v. First National Bank, 65 Modern Law Review, n. ${ }^{\circ}$ 5, 763-773 (2002). https://www.jstor.org/s table/1097615

Ewoud Hondius, Unfair Contract Terms: Towards a European Law Introduction, 5 European Review of Private Law, n. ${ }^{\circ}$ 2, 121-134 (1997). https://kluwerlawonline.com/journalarticle/European+Review+of+Private+Law/5.2/ 160150

François-Xavier Licari, Art. 1162 à 1171 Fasc. 50 : Déséquilibre significatif du contrat, Juris-Classeur Périodique (La Semaine juridique), 23 (2017).

Gerhard Dannemann, Comparative Law: Study of Similarities or Differences, en The Oxford Handbook of Comparative Law, 383-419 (Mathias Reimann and Reinhard Zimmermann, eds., Oxford University Press, 2006). https://do i.org/10.1093/oxfordhb/9780199296064.013.0012

Gesetz über Fernabsatzverträge und andere Fragen des Verbraucherrechts sowie zur Umstellung von Vorschriften auf Euro. Bundesgesetzblatt $n^{\circ} 28,27$ de junio de 2000. (Alemania).

Gesetz zur Änderung des AGB-Gesetzes und der Insolvenzordnung. Bundesgesetzblatt no 36, 19 de julio de 1996 (Alemania).

Gesetz zur Modernisierung des Schuldrechts. Bundesgesetzblatt nº 61, 26 de noviembre de 2001 (Alemania).

Gesetz zur Regelung des Rechts der Allgemeinen Geschäftsbedingungen (AGB-Gesetz). Bundesgesetzblatt n. ${ }^{\circ}$ 141, 9 de diciembre de 1976 (Alemania).

Gilles Paisant, Propositions pour une réforme du droit des clauses abusives (après la directive du 5 avril 1993), JCPG (1994)

Guy Raymond, Juris Classeur Concurrence - Consommation, Fasc. 820 : Clauses Abusives, (2018). 
Hans-W. Micklitz, Do Consumers and Businesses Need a New Architecture of Consumer Law? A Thought Provoking Impulse, 32 Yearbook of European Law, n. ${ }^{\circ}$ 1, 266-367 (2013). https://doi.org/10.1093/yel/yet006

Hugh Beale, English Law Reform and the Impact of European Private Law, en The Harmonisation of European Contract Law: Implications for European Private Laws, 31-38 (Stefan Vogenauer \& Stephen Weatherill eds., Hart Publishing, 2006). https://doi.org/10.5040/9781472559845.ch-003

Hugh Beale, Unfair Terms in Contracts: Proposals for Reform in the UK, Journal of Consumer Policy, n. ${ }^{\circ} 27,289-316$ (2004). https://doi.org/10.1023/B:COPO.0000040515.67198.1d

III Christian Larroumet \& Sarah Bros, Traité de droit Civil, - Les obligations - Le contrat (Economica, 2018).

J. Ghestin \& I. Marchessaux-Van Melle, L'application en France de la directive visant à éliminer les clauses abusives après l'adoption de la loi n. ${ }^{\circ} 95-96$ du ler février 1995, 31 Entreprise et Affaires, n. ํ 3, 481 (1995).

Jean-Christophe Grall \& Guillaume Mallen, Le déséquilibre significatif en droit français : une notion, deux textes, deux régimes juridiques, Grall \& Associés, (2017). https://www.grall-legal.fr/fr/desequilibre-significatif-en-droit-fra ncais/\#.XP-tqIhKjIV

Jérôme Huet, Propos amers sur la directive du 5 avril 1993 relative aux clauses abusives, La Semaine Jiridique, n. ${ }^{\circ}$ 1, 309 (1994).

Klaus Jochen Albiez Dohrmann, Un nuevo derecho de obligaciones. La Reforma 2002 del BGB, 55 Anuario de Derecho Civil, n. ${ }^{\circ}$ 3, 1133-1227 (2002). https://dialnet.unirioja.es/descarga/articulo/303664.pdf

Konrad Zweigert \& Hein Kötz, An Introduction to Comparative Law (3.. ed, Oxford University Press, 1998).

$\mathrm{La}$ Lettre des Réseaux - Déséquilibre significatif (N. ${ }^{\circ}$ Spécial - Octobre 2017) (Francia). https://www.lettredesreseaux.com/P-2305-451-A1-desequilibre-significatif-article-442-6,-i,-2-ducode-de-commerce.html

Lars Leuschner, "AGB-Recht für Verträge zwischen Unternehmen" Unter besonderer Berücksichtigung von Haftungsbeschränkungen. Informe encargado por Bundesministeriums der Justiz und für Verbraucherschutz (2014). http://www.bmjv.de/SharedDocs/Downloads/DE/Fachinformationen/Abschlussbericht-AGB-Forsc hungsprojekt.pdf?_blob=publicationFile

Loi n. ${ }^{\circ}$ 2008-776. De modernisation de l'économie. 4 de agosto de 2008 (Francia).

Loi n. ${ }^{\circ}$ 2014-344. Relative à la consommation. 17 de marzo de 2014 (Francia).

Loi n. ${ }^{\circ}$ 2017-203. Ratifiant les ordonnances n²016-301 du 14 mars 2016 relative à la partie législative du code de la consommation et $\mathrm{n}^{\circ} 2016-351$ du 25 mars 2016 sur les contrats de crédit aux consommateurs relatifs aux biens immobiliers à usage d'habitation et simplifiant le dispositif de mise en œuvre des obligations en matière de conformité et de sécurité des produits et services. 21 de febrero 2017 (Francia).

Loi.$^{\circ}$ 2018-287. Ratifiant l'ordonnance $n^{\circ} 2016-131$ du 10 février 2016 portant réforme du droit des contrats, du régime général et de la preuve des obligations. 20 de abril de 2018 (Francia).

Loi n. ${ }^{\circ}$ 95-96. Concernant les clauses abusives et la présentation des contrats et régissant diverses activités d'ordre économique et commercial. 1 de febrero de 1995 (Francia).

Louis Vogel \& Joseph Vogel, Le déséquilibre significatif, Dossier Concurrence, Distribution, Consommation (2.. ed., Bruylant, 2017).

M. Ancel. Utilité et methodes du droit comparé. Eléments d'introduction générale à l'étude comparative des droit, 23 Revue internationale de droit comparé, n. ${ }^{\circ} 4,933-935$ (1971). https://www.persee.fr/doc/ridc_0035-3337_19 71_num_23_4_16125

Maria Elisa Morales, Control preventivo de cláusulas abusivas (Ediciones Der, 2018).

Maria Elisa Morales, La reforma del Derecho inglés en materia de cláusulas abusivas, Revista de Derecho Privado, n. ${ }^{\circ} 28$, 281-304 (2017). http://dx.doi.org/10.4067/s0718-80722017000100281

Maria Luisa Vives, Traducción de la reforma 2002 del BGB, 55 Anuario de Derecho Civil, n. 3, 1229-1310 (2002).

María Natalia Mato, El control de contenido en la contratación mediante condiciones generales entre empresarios en el Derecho Comparado y Europeo, 7 Cuadernos de Derecho Transnacional, n. ${ }^{\circ} 2,216-282$ (2015). https://e-revist as.uc3m.es/index.php/CDT/article/view/2784 
Martine Béhar-Touchais, Que penser de l'introduction d'une protection contre les clauses abusives dans le code de commerce? Revue des contrats, n. ${ }^{\circ} 3,1258$ (2009).

Matthias Lehmann \& Johannes Ungerer, Differenzierte Unternehmerbegriffe und differenzierte Missbräuchlichkeitsanforderungen innerhalb der B2B-Inhaltskontrolle, en Standardisierte Verträge-zwischen Privatautonomie und rechtlicher Kontrolle, 295-312 (Johann Kindl, María Pilar Perales Viscasillas \& Tatiana Arroyo eds., Standardisierte Verträge-zwischen Privatautonomie und rechtlicher Kontrolle, 2017).

Ordonnance n. ${ }^{\circ}$ 2016-131. Portant réforme du droit des contrats, du régime général et de la preuve des obligations. 10 de febrero de 2016 (Francia).

Ordonnances n. ${ }^{\circ}$ 2016-301. Relative à la partie législative du code de la consommation. 14 de marzo de 2016 (Francia).

Ordonnances n..$^{\circ}$ 2016-351. Sur les contrats de crédit aux consommateurs relatifs aux biens immobiliers à usage d'habitation et simplifiant le dispositif de mise en œuvre des obligations en matière de conformité et de sécurité des produits et services. 25 de marzo de 2016 (Francia).

Reinhard Zimmermann, Consumer Contract Law and General Contract Law: The German Experience, 58 Current Legal Problems, n. ${ }^{\circ}$ 1, 415-489 (2005). http://hdl.handle.net/11858/00-001M-0000-0019-C489-0

Sander Van Loock, Unfair Terms in Contracts Between Businesses. A Comparative Overview in Light of the Common European Sales Law, en The Position of Small and Medium-Sized Enterprises in European Contract Law, 83-135 (Marco B. M. Loos \& Ilse Samoy eds., Intersentia, 2014).

Stéphane S. Piédelièvre, Droit de la Consommation (2. ${ }^{a}$ ed., 2014).

Stéphanie Porchy-Simon, Droit Civil $2^{\mathrm{e}}$ année, Les Obligations (Dalloz, 2018).

The Unfair Terms in Consumer Contracts Regulations 1994. № 3159. TSO. 1 de julio de 1995 (Reino Unido).

Tim Drygala, Unfair contract terms in business to business transactions, en Private Autonomy in Germany and Poland and in the Common European Sales Law, 35-52 (Tim Drygala et al. eds., Sellier European Law Publishers, 2012). https://doi.org/10.1515/9783866539631

Unfair Contract Terms Act 1977. HMSO. 26 de noviembre 1977. (Reino Unido).

Unión Europea, Directiva 2002/65/CE del Parlamento Europeo y del Consejo, relativa a la comercialización a distancia de servicios financieros destinados a los consumidores, y por la que se modifican la Directiva, 90/619/CEE del Consejo y las Directivas 97/7/CE y 98/27/CE (23 de septiembre, 2002).

Unión Europea, Directiva 93/13/CEE del Consejo, sobre las cláusulas abusivas en los contratos celebrados con consumidores ( 5 de abril, 1993).

Werner Müller, AGB-Kontrolle auf dem Prüfstand. Für Eine Lockerung Der Agb-Kontrolle Im Unternehmerischen Geschäftsverkeh, IWRZ- Zeitschrift für Internationales Wirtschaftsrecht, n. ${ }^{\circ}$ 4, 153-160, (2018).

Xavier Henry, Clauses abusives dans les contrats commerciaux : état des lieux dix ans après, AJ Contrat, 370-376 (2018). http://avocats-h.com/wp-content/uploads/2018/09/Clauses-abusives-dans-les-contrats-commerciau x-Etat-des-lieux-dix-ans-apr\%C3\%A8s-Dalloz-AJ-Contrat-\%E2\%80\%93-ao\%C3\%BBt-septembre-2018-\%E2 \%80\%93-n\%C2\%B0-8-9-p.-370..pdf

Yann Utzschneider \& Alexandra Lamothe, Que penser d'une règle de protection contre les clauses abusives dans le Code de commerce? Revue des contrats, n. ${ }^{\circ}$ 3, 1261 (2009).

Yves Picod, Droit du marché et droit commun des obligations. RTDCom. Revue trimestrielle de droit commercial et de droit économique (1998). https://halshs.archives-ouvertes.fr/halshs-02251140

\section{Notas}

* Artículo de investigación. Se enmarca en el proyecto FONDECYT Iniciación N. 11190543 titulado "Criterios de verificación de asimetría en las relaciones B2B. Una perspectiva de Derecho Comparado".

1 Sander Van Loock, Unfair Terms in Contracts Between Businesses. A Comparative Overview in Light of the Common European Sales Law, en The Position of Small and Medium-Sized Enterprises in European Contract Law, 83-135 (Marco B. M. Loos \& Ilse Samoy eds., Intersentia, 2014). 

consumidores ( 5 de abril, 1993).

11 Hugh Beale, English Law Reform and the Impact of European Private Law, en The Harmonisation of European Contract Law: Implications for European Private Laws, 31 (Stefan Vogenauer \& Stephen Weatherill eds., Hart Publishing, 2006

12 Parte de estas críticas se pueden revisar en: Ewoud Hondius, Unfair Contract Terms: Towards a European Law Introduction, 5 European Review of Private Law, n. ${ }^{\circ}$ 2, 134 (1997); Hugh Beale, Unfair Terms in Contracts: Proposals for Reform in the UK, Journal of Consumer Policy, n. ${ }^{\circ}$ 27, 289-316 (2004); Hugh Beale, English Law Reform and the Impact of European Private Law, op. cit.

13 "[P]ossibly the single most significant piece of legislation in the field of contract law". Elizabeth Macdonald, Scope and Fairness of the Unfair Terms in Consumer Contracts Regulations: Director General of Fair-Tradingv. First National Bank, 65 Modern Law Review, n. ${ }^{\circ}$, 763(2002).

14 Consumer Rights Act. 2015 c. 15. TSO. 26 marzo 2015 (Reino Unido).

15 Consumer Rights Act. 2015 c. 15. TSO. 26 marzo 2015 (Reino Unido). Sección 62. Cláusula abusiva se define como aquella que, siendo contraria a los requerimientos de la buena fe, causa un significativo desequilibrio entre los derechos de las partes contratantes en detrimento del consumidor.

16 El tribunal debe tener en cuenta las específicas circunstancias existentes al momento de celebrar el contrato, las otras cláusulas del contrato y la naturaleza del objeto principal del contrato. Este es el test de abusividad.

17 Consumer Rights Act. 2015 c. 15. TSO. 26 marzo 2015 (Reino Unido), anexo 2, parte 1, pa\#rrafos 5, 12 y 14.

18 Se trata de la implementación de art. 15 de la Directiva 2002/65/CE del Parlamento Europeo y del Consejo, de 23 de septiembre de 2002, relativa a la comercialización a distancia de servicios financieros destinados a los consumidores, y por la que se modifican la Directiva 90/619/CEE del Consejo y las Directivas 97/7/CE y 98/27/CE, según el cual será abusiva toda cláusula contractual o condición que ponga sobre el consumidor la carga de probar el cumplimiento de la totalidad o parte de las obligaciones que le incumben al proveedor en virtud de dicha directiva CRA, parte 2, seccio\#n 63(6) y (7), que implementa el art. 15 directiva 2002/65/CE del Parlamento europeo y del Consejo de 23 de septiembre de 2002 relativa a la comercialización a distancia de servicios financieros destinados a los consumidores, y por la que se modifican la directiva 90/619/CEE del Consejo y las directivas 97/7/CE y 98/27/CE. Consumer Rights Act. 2015 c. 15. TSO. 26 marzo 2015 (Reino Unido), parte 2, secciones 65 y 66.

19 Por último, las cláusulas que excluyen o restringen la responsabilidad por muerte o lesiones personales por negligencia del proveedor están prohibidas y no producen efecto alguno $110 \mathrm{CRA}$, parte 2 , secciones 65 y 66 .

20 De conformidad a la directiva 93/13, se prevén algunas excepciones a la evaluación de equidad o justicia de una cla\# usula (exclusion from assessment of fairness). Se trata de las cla\#usulas que especifican el objeto principal del contrato y las que se refieren a la adecuacio\#n del precio. Lo interesante es que CRA precisa estas excepciones e incorpora un te\#rmino nuevo en el a\#mbito de la regulacio\#n de cla\#usulas abusivas en el Derecho ingle\#s, cual es prominent, te\# $\mathrm{r}$ - mino cuya traduccio\#n ma\#s adecuada, ya empleada en el apartado anterior, es la de "notorio(a)" dado que se refiere a que\# tan visible o evidente debe ser la cla\#usula.

21 Consumer Rights Act. 2015 c. 15. TSO. 26 marzo 2015 (Reino Unido). Parte 2, sección 76(2) y Parte 1, sección 2(2) (3).

22 La estructura de las leyes en el Reino Unido suele ser así porque, como se sabe, el Reino Unido, aunque es un Estado unitario, está conformado por Inglaterra, Gales, Escocia e Irlanda del Norte. En un sentido técnico, cuando se habla de derecho inglés, en cuanto a su ámbito de validez territorial, se incluye a Inglaterra y Gales. En consecuencia, no quedan comprendidos Irlanda del Norte ni Escocia. Por lo tanto, el derecho inglés técnicamente no se está haciendo alusión al derecho de todo el Reino Unido. Estas distinciones suelen reflejarse en la estructura de las leyes.

23 Sección 11(2) en relación con Schedule 2, UCTA.

24 La tercera parte se refiere a los contratos de suministro internacional, los cuales se encuentran excluidos del control de abusividad. 
6 en Standardisierte Verträge - zwischen Privatautonomie und rechtlicher Kontrolle, 237-258 (Johann Kindl, María Pilar Perales Viscasillas \& Tatiana Arroyo eds., Standardisierte Verträge-zwischen Privatautonomie ${ }^{\text {xix }}$ und rechtlicher Kontrolle, 2017).

27 Reinhard Zimmermann, Consumer Contract Law and General Contract Law: The German Experience, 58 Current Legal Problems, n. ${ }^{\circ}$ 1, 415-489 (2005).

28 S138 Sittenwidriges Rechtsgeschäft; Wucher (1) Ein Rechtsgeschäft, das gegen die guten Sitten verstößt, ist nichtig.

29 En este apartado, cualquier referencia a un artículo sin especificación se entiende hecha al BGB.

30 Reinhard Zimmermann, op. cit., 433. Véase jursiprudencia al respecto en B. Gsell, op cit., ártículo 242.

31 Reinhard Zimmermann, op. cit., 441.

32 Klaus Jochen Albiez Dohrmann, Un nuevo derecho de obligaciones. La Reforma 2002 del BGB, 55 Anuario de Derecho Civil, n. ${ }^{\circ}$ 3, 1144 (2002).

33 Ley de modernización del Derecho de obligaciones de 26 de noviembre de 2001 (Gesetz zur Modernisierung des Schuldrechts), que entró en vigor el 1 de enero de 2002. Gesetz zur Modernisierung des Schuldrechts. Bundesgesetzblatt no 61, 26 de noviembre de 2001 (Alemania).

34 Decisión que no estuvo exenta de polémica, como explica Klaus Jochen Albiez Dohrmann, op. cit., 1148. “[d]espués del BGB, según muchos, la AGB-G ha sido el mejor logro de la cultura iusprivatista alemana ¿Por qué había que romper este edificio tan bien diseñado?”. Del mismo autor, véase listado de leyes especiales sobre derecho de consumo también incorporadas en otros artículos del BGB.

35 En lo sucesivo seguiremos la traducción de los artículos 305-310 del BGB de Maria Luisa Vives, Traducción de la reforma 2002 del BGB, 55 Anuario de Derecho Civil, n. 3, 1229-1310 (2002).

36 Como señalamos, esta incorporación fue polémica. Así, en opinión de Reinhard Zimmermann, op. cit., 459, “[a]s a result, the new $\$ \$ 305-310$ seem like a piece of pop music tossed into the second movement of a classical symphony: a corpus alienum without intellectual connection to its surroundings. The incorporation of the Standard Terms of Business Act into the BGB, in other words, has been a purely formal exercise; it has not led to anything that could be called an integration into the fabric of the BGB"

37 Reinhard Zimmermann, op. cit., 434. Aunque no estuvo fuera de polémicas, pues como explica B. Gsell, op cit., 240. Previamente a la codificación en la AGBG, se genera el acalorado debate acerca de la conveniencia de establecer un régimen de protección amplio o solo limitado al consumidor propiamente tal.

38 Artículo 13.

39 Artículo 14.

40 María Natalia Mato, El control de contenido en la contratación mediante condiciones generales entre empresarios en el Derecho Comparado y Europeo, 7 Cuadernos de Derecho Transnacional, n. ${ }^{\circ}$ 2, 216-282 (2015); Matthias Lehmann \& Johannes Ungerer, Differenzierte Unternehmerbegriffe und differenzierte Missbräuchlichkeitsanforderungen innerhalb der B2B-Inhaltskontrolle, en Standardisierte Verträge-zwischen Privatautonomie und rechtlicher Kontrolle, 295-312 (Johann Kindl, María Pilar Perales Viscasillas \& Tatiana Arroyo eds., Standardisierte Verträge-zwischen Privatautonomie und rechtlicher Kontrolle, 2017).

41 Tim Drygala, Unfair contract terms in business to business transactions, en Private Autonomy in Germany and Poland and in the Common European Sales Law, 44 (Tim Drygala et al. eds., Sellier European Law Publishers, 2012).

42 Christoph Schmith \& Detlef Ulmer, Allgemeine Geschäftsbedingungen und Verträge für Unternehmen: Chancen und Risiken, 36 (Springer, 2010).

43 Véase sentencias en dicho sentido en Lars Leuschner, "AGB-Recht für Verträge zwischen Unternehmen" Unter besonderer Berücksichtigung von Haftungsbeschränkungen. Informe encargado por Bundesministeriums der Justiz und für Verbraucherschutz (2014); María Natalia Mato, op. cit., 254-258. Así, explica la mencionada autora, "[d]ado que la transparencia, como hemos señalado, está íntimamente vinculada con la capacidad de comprensión del adherente, parece evidente que la experiencia comercial posibilita a un empresario tener unas mayores posibilidades de conocimiento y una mayor información. Por lo tanto, los requisitos del mandato de transparencia deben ser adaptados a los horizontes empresariales implicando, en general, una relajación de los mismos, sin que pueda ser aplicado el principio con la misma intensidad como en el ámbito de la contratación con consumidores".

44 Véase sentencias en Christoph Schmith \& Detlef Ulmer, op. cit., 37-39; María Natalia Mato, op. cit., 258-261 (2015); Werner Müller, AGB-Kontrolle auf dem Prüfstand. Für Eine Lockerung Der Agb-Kontrolle Im Unternehmerischen Geschäfsverkeh, IWRZ- Zeitschrift für Internationales Wirtschaftsrecht, n. ${ }^{\circ}$ 4, 155, (2018).

45 "Wenn eine Klausel in Allgemeinen Geschäftsbedingungen bei ihrer Verwendung gegenüber Verbrauchern unter die Verbotsnormen des $₫ 309$ BGB fällt, sei dies ein Indiz dafür, dass sie auch im Falle der Verwendung gegenüber Unternehmern zu einer unangemessenen Benachteiligung führen kann, es sei denn, sie kann wegen der besonderen 
Interessen und Bedürfnisse des unternehmerischen Geschäftsverkehrs ausnahmsweise als angemessen angesehen warden”. Christoph Schmith \& Detlef Ulmer, op. cit., 37. Véase también, B. Gsell, op cit. 242-243.

46 Matthias Lehmann \& Johannes Ungerer, op. cit. Como explican los mencionados autores, "Zu Beginn stehen die „kleinsten“ oder „jüngsten“ Unternehmer, auf die am meisten Rücksicht genommen wird. Die nachfolgenden Kategorien betreffen größere Unternehmen, denen aber immer noch ein besonderer Schutz zuteil wird. Am Ende der Kategorisierung lassen sich die Großunternehmen verorten, die keinen Vorzug innerhalb der Inhaltskontrolle mehr genießen”. De todas formas, no significa que las grandes empresas no estén sujetas eventualmente a un control de contenido, sólo que no reciben protección especial. Íd., 305-306.

47 Debate que comienza a tomar fuerza como consecuencia de la elaboración del Borrador de Marco Común de Referencia en Europa (Draft Common Frame of Reference [DCFR]). Hans-W. Micklitz, Do Consumers and Businesses Need a New Architecture of Consumer Law? A Thought Provoking Impulse, 32 Yearbook of European Law, n. ${ }^{\circ}$ 1, 287 (2013).

48 B. Gsell, op cit., 246.

49 Sobre este punto, ver: Antonia Sommerfeld, Rechtsflucht ins Ausland wegen des deutschen AGB-Rechts im B2B-Verkehr, 11 RIW-Recht der internationalen Wirtschaft, n. ${ }^{\circ}$ 15, $741-747$ (2018). Destaca Hans-W. Micklitz, op. cit., 287: “[t] he arguments presented can be reduced to a common denominator. The AGB-control of $\mathrm{b} 2 \mathrm{~b}$ contracts practised by the courts created a Procrustean bed, from which companies could only escape by contracting under a foreign law, for example under Swiss law which to date does not include an AGB-control. The jurisprudence is blamed having needlessly transferred the AGBG, usually - though inaccurately - seen as having been designed as a law on consumer protection, to the field of $\mathrm{b} 2 \mathrm{~b}$. Only very occasionally are there voices that warn against a hasty change of the legal position. The heated discussion has led to the bon mot: 'All against Graf (Count) von Westphalen'”. Cabe destacar que Graf von Westphalen es uno de los referentes sobre AGB en Alemania y ha sido una de las voces más autorizadas en defensa del statu quo en esta materia.

50 Matthias Lehmann \& Johannes Ungerer, op. cit., 306-311.

51 Íd., 307-308.

52 Maria Elisa Morales, Control preventivo de cláusulas abusivas, 12-15 (Ediciones Der, 2018).

53 Claire-Marie Peglion-Zika, La notion de clause abusive au sens de l'article L. 132-1 du Code de la consommation (2013) (tesis doctoral, Université Panthéon-Assas). La autora cita como doctrina a favor: Gilles Paisant, Propositions pour une réforme du droit des clauses abusives (après la directive du 5 avril 1993), JCPG (1994); como doctrina en contra: Jérôme Huet, Propos amers sur la directive du 5 avril 1993 relative aux clauses abusives, La Semaine Jiridique, n. ${ }^{\circ}$ 1, 309 (1994).

54 "Y, en estas circunstancias, es evidente que no tiene la obligación legal de implementar en el derecho francés la Directiva de 5 de abril de 1993, sobre las cláusulas abusivas". Jérôme Huet, op. cit.

55 Commission des Clauses Abusives. Recommandation de synthèse n. ${ }^{\circ} 91-02$ relative à certaines clauses insérées dans les contrats conclus entre professionnels et non-professionnels ou consommateurs (BOCCRF du 6/09/1991).

56 J. Ghestin \& I. Marchessaux-Van Melle, L'application en France de la directive visant á éliminer les clauses abusives après l'adoption de la loi n. ${ }^{\circ} 95-96$ du ler février 1995, 31 Entreprise et Affaires, n. ${ }^{\circ}$ 3, 481 (1995).

57 "Las cláusulas contractuales que no se hayan negociado individualmente se considerarán abusivas si, pese a las exigencias de la buena fe, causan en detrimento del consumidor un desequilibrio importante entre los derechos y obligaciones de las partes que se derivan del contrato". Unión Europea, Directiva 93/13/CEE del Consejo, sobre las cláusulas abusivas en los contratos celebrados con consumidores ( 5 de abril, 1993). Artículo 3.1.

58 J. Ghestin \& I. Marchessaux-Van Melle, op. cit.

59 Se estima que, en la práctica, raramente la buena fe estará presente en un contrato que contiene una cláusula abusiva."Pourtant en pratique, la bonne foi sera rarement présente dans un contrat comportant une clause abusive", Stéphane S. Piédelièvre, Droit de la Consommation, 454 (2. a ed., 2014).

60 Loi n. ${ }^{\circ}$ 95-96. Concernant les clauses abusives et la présentation des contrats et régissant diverses activités d'ordre économique et commercial. 1 de febrero de 1995 (Francia). Para un análisis de la implementación ver: J. Ghestin \& I. Marchessaux-Van Melle, op. cit.

61 Stéphanie Porchy-Simon, Droit Civil $2^{\mathrm{e}}$ année, Les Obligations, 128 (Dalloz, 2018).

62 Para ser fieles a la nomenclatura francesa, y para evitar disquisiciones problemáticas de lenguaje, se sugiere revisar el art. preliminar del Code de la Consommation (modificado por la Loi n. ${ }^{\circ}$ 2014-344. Relative à la consommation. 17 de marzo de 2014 (Francia), posteriormente modificada por la Loi n. ${ }^{\circ} 2017-203$ du 21 février 2017 ratifiant les ordonnances $n$. 。 2016-301 du 14 mars 2016 relative à la partie législative du code de la consommation et n. $2016-351$ du 25 mars 2016 sur les contrats de crédit aux consommateurs relatifs aux biens immobiliers à usage d'habitation et simplifiant le dispositif de mise en œuvre des obligations en matière de conformité et de sécurité des produits et services). La norma conceptualiza al profesional, consumidor y no profesional. Es del caso hacer presente que la disposición en referencia viene a dar solución a las dificultades que se habían presentado en el derecho francés, en atención al sentido que debía atribuírsele a la palabra consumidor. En rigor, la Loi n. ${ }^{\circ}$ 95-96. Concernant les clauses abusives et la présentation des 
contrats et régissant diverses activités d'ordre économique et commercial. 1 de febrero de 1995, que integró al derecho francés la Directiva comunitaria n..$^{\circ}$ 93/13 de 5 de abril de 1993, concernant les clauses abusives dans les contrats conclus avec les consommateurs, prescindió del concepto de consumidor consignado por la misma. Sobre este punto, ver: Camille Dupin, La notion de consommateur, (2013-2014), (Mémoire Master Droit des Contrats, Université Pris), que recopila el desarrollo de la noción hasta el pronunciamiento legislativo.

63 Yves Picod, Droit du marché et droit commun des obligations. RTDCom. Revue trimestrielle de droit commercial et de droit économique (1998).

64 Loi n. 2008-776. De modernisation de l'économie. 4 de agosto de 2008 (Francia).

65 La disposición generó revuelo entre los comentaristas. En este sentido Yann Utzschneider \& Alexandra Lamothe, Que penser d'une règle de protection contre les clauses abusives dans le Code de commerce? Revue des contrats, n..$^{\circ}$ 3, 1261 (2009) enseñan que: "La nueva disposición, que es muy general, no establece ningún límite expreso en el control del juez de las disposiciones contractuales. Se refiere indiscriminadamente a cualquier "desequilibrio significativo en los derechos y obligaciones de las partes". Por lo tanto, en principio, están sujetos al control del juez de todos los tipos de cláusulas: las cláusulas de responsabilidad, garantía, cláusulas relativas al suministro, pedidos, entrega, etc. Por lo tanto, la nueva norma podría permitir al juez interferir en la ley de las partes, controlando el equilibrio contractual, desafiando el principio de la autonomía de la voluntad. El nuevo texto podría incluso permitir controlar, sobre la base de un "desequilibrio significativo", las cláusulas de precios y, en general, las obligaciones financieras estipuladas en el contrato”. En la misma línea, ver: Martine Béhar-Touchais, Quepenser de l'introduction d'une protection contre les clauses abusives dans le code de commerce? Revue des contrats, n. $^{\circ}$ 3, 1258 (2009).

66 Xavier Henry, Clauses abusives dans les contrats commerciaux : état des lieux dix ans après, AJ Contrat, 370-376 (2018).

67 En virtud de la Ordenanza n. ${ }^{\circ}$ 2016-131. Portant réforme du droit des contrats, du régime général et de la preuve des obligations. 10 de febrero de 2016 (Francia).

68 Son dos las vías a través de las cuales se puede tener una cláusula por no escrita: cláusulas abusivas declaradas por decreto, y cláusulas abusivas declaradas por el juez. Sobre este punto, puede verse Stéphanie Porchy-Simon, op. cit., 129.

69 Código de Consumo (Code de la Consommation), 13 de noviembre de 2020. Art. 241-1.

70 Fruto de la Loi n. ${ }^{\circ} 2018-287$ du 20 avril 2018 ratifiant l'ordonnance.$^{\circ}$ 2016-131 du 10 février 2016 portant réforme du droit des contrats, du régime général et de la preuve des obligations.

71 Cyril Grimaldi, Les limites à la libre détermination du contenu du contrat dans le nouveau droit des contrats, Lextenso étudiant, (10 de noviembre, 2016).

72 Guy Raymond, Juris Classeur Concurrence - Consommation, Fasc. 820 : Clauses Abusives, (2018).

73 El criterio amplio en el origen de la abusividad es también percibible en el derecho del consumo, el cual no la limita exclusivamente al contrato de adhesión. Así, ella puede también derivar de contratos libremente negociados, según se desprende del art. L. 212-1, del Código del Consumo.

74 Respecto de la indicada condición resulta relevante la sentencia Corte de Apelaciones de París, JurisData: 2017-010849. 17 de mayo de 2017 (Francia). La sentencia rechaza el cumplimiento del requisito de sometimiento o del intento de someter, puesto que dicha situación debe converger en vínculos asimétricos entre las partes del contrato, lo cual no fue estimado como efectivo.

75 La jurisprudencia ha estimado que una cláusula resolutoria puede significar un desequilibrio significativo, Así lo ha resuelto la Corte de Apelaciones de París en fallo de 30 de mayo de 2017, JurisData: 2017-010882 (Francia). Sin perjuicio de lo anterior, en la especie se resolvió en definitiva que no fue demostrado ningún desequilibrio patrimonial efectivo, solamente se limitó a reconocer que pueden existir cláusulas susceptibles de engendrar dicha asimetría.

76 Para un panorama jurisprudencial francés concerniente al desequilibrio significativo de los años 2016-2017, puede verse: La Lettre des Réseaux - Déséquilibre significatif (N. ${ }^{\circ}$ Spécial - Octobre 2017) (Francia). Con la misma finalidad, puede también verse: Louis Vogel \& Joseph Vogel, Le déséquilibre significatif, Dossier Concurrence, Distribution, Consommation (2. ${ }^{\text {a }}$ ed., Bruylant, 2017).

77 Se sugiere revisar, François-Xavier Licari, Art. 1162 à 1171 Fasc. 50 : Déséquilibre significatif du contrat, Juris-Classeur Périodique (La Semaine juridique), 23 (2017).

78 III Christian Larroumet \& Sarah Bros, Traité de droit Civil, —Les obligations - Le contrat, 410 (Economica, 2018).

79 Íd.

80 Jean-Christophe Grall \& Guillaume Mallen, Le déséquilibre significatif en droit français : une notion, deux textes, deux régimes juridiques, Grall \& Associés, (2017).

81 M. Ancel. Utilité et methodes du droit comparé. Eléments d'introduction générale à l'étude comparative des droit, 23 Revue internationale de droit comparé, n. ${ }^{\circ}$ 4, 933-935 (1971).

82 Gerhard Dannemann, op. cit., 406.

\section{Licencia Creative Commons CC BY 4.0}


María Elisa Morales Ortiz, et al. Cláusulas abusivas entre empresas. Evolución ...

Para citar este articulo/To cite this article: María Elisa Morales Ortiz, Pamela Andrea Mendoza Alonzo \& Renzo Esteban Munita Marambio, Cláusulas abusivas entre empresas. Evolución en los principales sistemas de derecho comparado, 69 Vniversitas (2020). https://doi.org//10.11144/Javeriana.vj69.caee 